\title{
Applying a participatory approach to the promotion of a culture of respect during childbirth
}

\author{
Hannah L. Ratcliffe ${ }^{1,2^{*}}$, David Sando ${ }^{1,3}$, Mary Mwanyika-Sando ${ }^{4}$, Guerino Chalamilla ${ }^{3,4^{\wedge}}$, Ana Langer ${ }^{1}$ \\ and Kathleen P. McDonald ${ }^{1,5}$
}

\begin{abstract}
Disrespect and abuse (D\&A) during facility-based childbirth is a topic of growing concern and attention globally. Several recent studies have sought to quantify the prevalence of D\&A, however little evidence exists about effective interventions to mitigate disrespect and abuse, and promote respectful maternity care. In an accompanying article, we describe the process of selecting, implementing, and evaluating a package of interventions designed to prevent and reduce disrespect and abuse in a large urban hospital in Tanzania. Though that study was not powered to detect a definitive impact on reducing D\&A, the results showed important changes in intermediate outcomes associated with this goal. In this commentary, we describe the factors that enabled this effect, especially the participatory approach we adopted to engage key stakeholders throughout the planning and implementation of the program. Based on our experience and findings, we conclude that a visible, sustained, and participatory intervention process; committed facility leadership; management support; and staff engagement throughout the project contributed to a marked change in the culture of the hospital to one that values and promotes respectful maternity care. For these changes to translate into dignified care during childbirth for all women in a sustainable fashion, institutional commitment to providing the necessary resources and staff will be needed.
\end{abstract}

Keywords: Maternal health, Participatory dissemination, Disrespect and abuse, Respectful maternity care, Tanzania, Health Workers for Change

\section{Background}

In an accompanying research article, Mitigating disrespect and abuse during childbirth in Tanzania: an exploratory study of the effects of two facility-based interventions in a large public hospital, we describe the process of selecting, implementing, and evaluating a package of interventions whose ultimate goal was to prevent and reduce disrespect and abuse (D\&A) during childbirth [1]. The study was conducted in an urban regional referral hospital in Dar es Salaam with a catchment area of 1.4 million people. The maternity section of the hospital serves as a referral site to over 40 lower level health facilities, and on average during

\footnotetext{
* Correspondence: hannah.ratcliffe@mail.harvard.edu

Deceased

${ }^{1}$ Women and Health Initiative, Department of Global Health and Population, Harvard T.H Chan School of Public Health, Boston, MA, USA

${ }^{2}$ Ariadne Labs at Brigham and Women's Hospital and the Harvard T.H. Chan

School of Public Health, Boston, MA, USA

Full list of author information is available at the end of the article
}

the study period there were 2060 deliveries, 311 maternal complications, 3 maternal deaths, and 68 neonatal deaths per month. (Facility data) While the study lacked the needed statistical power to definitively measure impact on disrespect and abuse, evidence presented in this commentary suggests that the changes introduced in the facility's culture have likely decreased the incidence of disrespect and abuse [2]. Here, we describe the evidence and enabling environment that we believe facilitated this change.

\section{Engaging providers and policymakers to foster collaboration}

Generating an open dialogue on disrespect and abuse in a healthcare setting can be challenging. Healthcare administrators, providers, and women seeking care are understandably apprehensive about identifying incidence of D\&A within their facility. In an effort to assuage any potential uneasiness towards conducting research around this sensitive 
area, the study team strategically engaged with key influencers at the facility, district and national levels of the health care system throughout the course of the project. These stakeholders included influential policymakers within the Ministry of Health and Social Welfare who had expressed support for this work in prior conversations with the study team, and leaders of the district-level Council Health Management Team responsible for the facility. We also engaged with key members of the study facility, i.e., nurses and midwives on the frontline of providing antenatal care services and influential members of hospital staff including the matron in-charge, head of obstetrics and gynecology, nurse incharge of the maternity ward, and the Director of Programs. We posit that the multilevel support generated through our participatory implementation process amplified the effect of the discrete interventions, reinforced the principles of respectful maternity care [3], and had a positive influence on the outcomes observed. Similar to prominent efforts aimed at reducing HIV stigmatization, including programs targeting the prevention of vertical HIV transmission during pregnancy [4], identifying D\&A during childbirth at the facility as an issue to be "corrected" and conducting a highly visible project were interventions in and of themselves.

\section{Sensitization}

At the project's initiation, a series of sensitization meetings were held with policymakers at the Tanzanian Ministry of Health and Social Welfare (MOHSW); the municipal medical officer of health; the Council Health Management Team (CHMT); and hospital administrators and health care providers in the maternity departments of the study hospital. These consultations focused on creating awareness of D\&A and generating support to further explore the issue from key maternal health stakeholders at the national, regional and municipal levels. We also consulted with members of the Staha study team, who at this time had initiated a similar study in the Tanga Region of Tanzania [5]. Our ability to engage the MOHSW and increase awareness of D\&A was greatly facilitated by this complementary work.

\section{Participatory dissemination}

In the first phase of the project, a baseline assessment found high levels of disrespect and abuse, both as reported by women and as directly observed by trained data collectors [6]. After the baseline assessment was completed, we conducted a three-step participatory dissemination process, the goal of which was to create a culture of transparency, collaboration, and institutional accountability and responsibility. Baseline results were presented initially to the hospital management team and then to providers at the study facility to allow them to internalize and reflect on the findings without assigning blame or attributing fault to specific individuals or areas of the hospital. The second dissemination meeting included hospital providers as well as district-level officials, and focused on receiving input about the feasibility, desirability, and sustainability of potential evidence-based interventions developed from an extensive literature review [7]. After the second consultation, a technical working group-comprised of maternity ward healthcare providers, hospital management representatives, municipal-level health managers, representatives from local and international partner organizations, and study team members-was formed to continue developing interventions that were deemed applicable and appropriate for the study facility. Finally, results were disseminated nationally to obtain feedback and generate high-level consensus on intervention selection. Participants included national representatives from the Ministry of Health; leaders from the regional and municipal health offices; maternal health experts from development partners; academics from medical and nursing institutions; representatives from medical and nursing professional associations; and health care providers and management from the study hospital.

Women who shared their experiences of disrespect and abuse during baseline data collection did not participate in the dissemination process. The decision to limit the audience to those working in the health system was based on the recommendations and requests from key influencers in the region. The rationale was that the initial identification and targeted reduction of D\&A needed to be provider-led to allow for acceptability, cooperation, and receptivity of facility-level interventions. Sensitivity around the issue of D\&A and tenuous patient-provider relationships-as evidenced by the baseline findings-could have escalated into disagreements and blaming exercises, and negated efforts to mitigate D\&A during childbirth.

\section{Intervention selection}

Based on this multi-level participatory process, two interventions were selected: Open Birth Days (OBD) and Respectful Maternity Care Workshops. Open Birth Days included a participatory health education session and tour of the study facility and were open to all women attending antenatal care at the facility during their third trimester. The sessions were designed to improve women's comfort with the facility and increase birth preparedness by facilitating communication with providers and providing a step-by-step guide to what to expect when they arrive at the facility for delivery. The Respectful Maternity Care Workshop consisted of six modules adapted from the Health Workers for Change curriculum [8]. The goals of the Workshop-which was facilitated by seasoned trainers and respected medical school professors-were for providers to reexamine how their 
current practice matches their professional codes of conduct, reflect on the needs and preferences of patients, and openly and honestly discuss the barriers that prevent the provision of RMC at the study facility. At the conclusion of the workshop, participants decided upon an action plan that could be carried out of their own accord with minimal influx of external resources. Steps taken by the facility through this action plan included improved use of privacy curtains, changes to staffing structure, and improvements in the speed of overtime payments, among others.

Rollout of the selected interventions began in April 2014 and continued through October 2014. Reflecting the success of the participatory selection process, nurses in the antenatal ward at the facility began OBD in December 2013 immediately after the national dissemination meeting using their own time, resources, and ideas to drive implementation. Beginning in April, these processes were standardized for implementation and evaluation, and the significant support from facility staff continued throughout the implementation period. The accompanying research article describes in detail the complete implementation and evaluation protocol, and provides evidence from routine monitoring and evaluation activities of marked progress towards achieving proximal and intermediary outcomes targeted under the project's theory of change (Fig. 1) [1]. In total, 362 women participated in the Open Birth Days Sessions (100 \% acceptance rate) and 76 staff from the maternity ward participated in the RMC Workshop (86 \% participation rate). Evidence from multiple monitoring tools shows that, over the course of the intervention, there were improvements in both proximal and distal outcomes including: patient knowledge of their rights and birth preparedness, provider knowledge of patient rights, provider attitudes, provider job satisfaction, and patient-provider communications and interactions. Additionally there is evidence that patients were more empowered after participation in an Open Birth Day session, and patient satisfaction with their delivery experience improved substantially from baseline $(75.8 \%$ very satisfied compared to $12.9 \%$ at baseline).

\section{Evidence of a substantial decrease in incidence of disrespect and abuse}

In addition to the successful achievement of intermediate outcomes noted in the accompanying research article, all available evidence points towards a declining trend in D\&A incidence, both as reported by women and as recorded by trained observers [1]. Among women interviewed in their homes four to six weeks after delivery at baseline, $70 \%$ reported experiencing any form of disrespect and abuse. At the time of evaluation, $18 \%$ of women interviewed four to six week post-delivery reported experiencing any form of D\&A (Table 1). Furthermore, reports of each category of disrespect and abuse also declined dramatically compared to baseline. For example, physical abuse, non-confidential care, lack of privacy, and nondignified care were reported by more than $50 \%$ of respondents at baseline, compared to less than $5 \%$ after the intervention was concluded.

Results from the direct observations of patientprovider interactions also show marked declines in D\&A, substantiating patient reports (Table 2). No examples of non-consented care were recorded during the evaluation, compared with incidences as high as

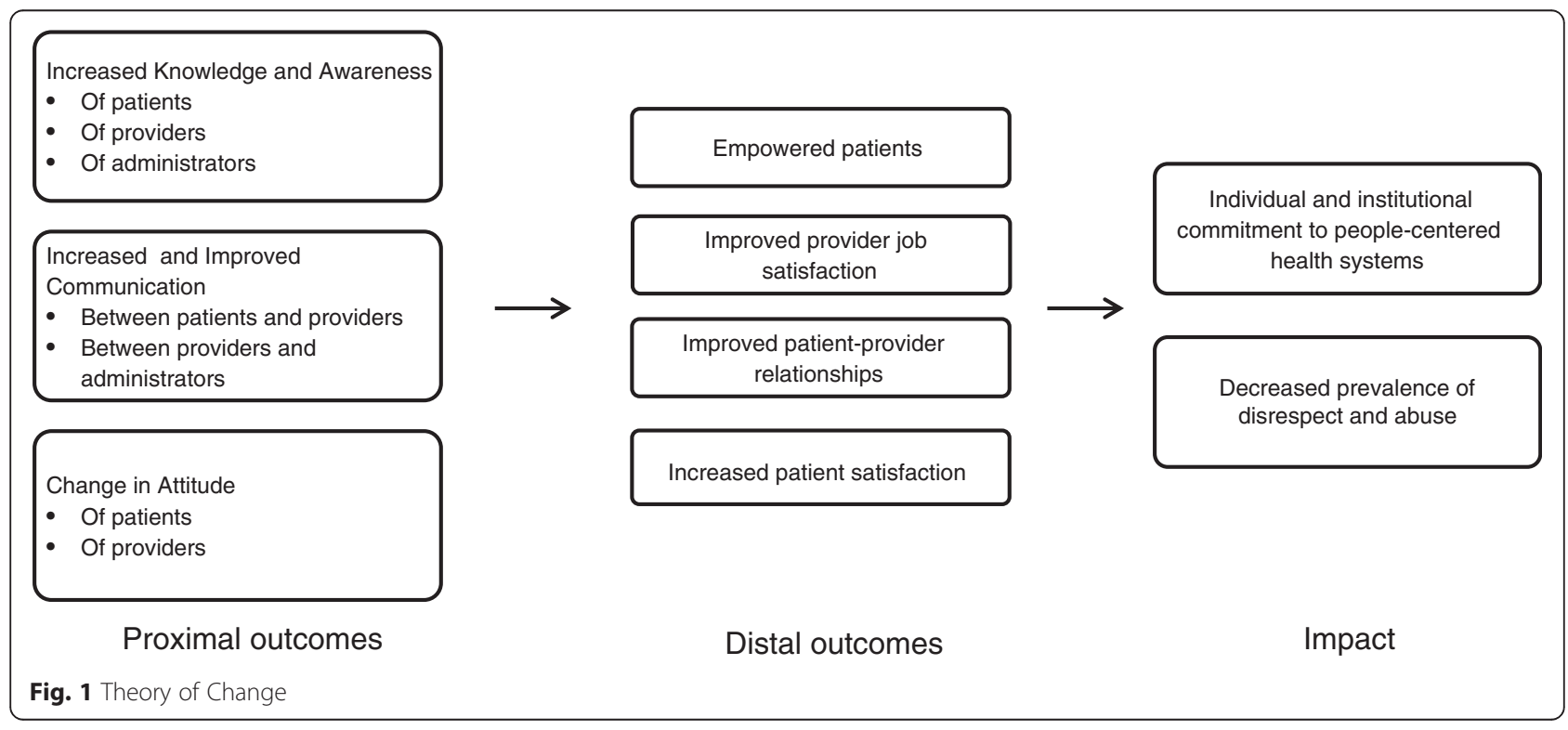


Table 1 Patient reported experiences of disrespect and abuse

\begin{tabular}{|c|c|c|c|c|}
\hline Type of Disrespect and Abuse & $\begin{array}{l}\text { Baseline } \\
\text { Exit Interview } \\
N=1914 \\
n(\%)\end{array}$ & $\begin{array}{l}\text { Baseline } \\
\text { CFU } \\
N=64 \\
n(\%)\end{array}$ & $\begin{array}{l}\text { Evaluation } \\
\text { CFU } \\
N=149 \\
n(\%)\end{array}$ & $\begin{array}{l}\text { Difference between } \\
\text { Evaluation and } \\
\text { Baseline CFU } \\
\%\end{array}$ \\
\hline Any form of disrespect or abuse & $278(15)$ & $50(70)$ & $26(18)$ & -52 \\
\hline Physical abuse & $84(5)$ & $33(52)$ & $2(1)$ & -51 \\
\hline Kicked & $2(0.1)$ & $1(2)$ & $0(0)$ & -2 \\
\hline Pinched & $22(1)$ & $3(5)$ & $0(0)$ & -5 \\
\hline Slapped & $23(1)$ & $17(27)$ & $1(1)$ & -26 \\
\hline Pushed & $12(0.6)$ & $4(6)$ & $0(0)$ & -6 \\
\hline Beaten & $4(0.2)$ & $5(8)$ & $0(0)$ & -8 \\
\hline Episiotomy without anesthesia & $1(0.1)$ & $1(2)$ & $0(0)$ & -2 \\
\hline Tied to the delivery bed & $2(0.1)$ & $0(0)$ & $0(0)$ & 0 \\
\hline Other & $17(0.9)$ & $9(14)$ & $1(1)$ & -13 \\
\hline Non-consented care & $4(0.2)$ & $3(5)$ & $1(1)$ & -4 \\
\hline Abdominal Palpation & $2(0.1)$ & $0(0)$ & $0(0)$ & 0 \\
\hline Vaginal Examination & $4(0.2)$ & $1(2)$ & $0(0)$ & -2 \\
\hline Episiotomy & $0(0)$ & $1(2)$ & $0(0)$ & -2 \\
\hline Other & $0(0)$ & $1(2)$ & $1(1)$ & -1 \\
\hline Non-confidential care & $32(2)$ & $34(54)$ & $2(1)$ & -53 \\
\hline Discussed personal issues in earshot of other clients & $1(0.1)$ & $1(2)$ & $0(0)$ & -2 \\
\hline Health information discussed with non-health staff & $0(0)$ & $2(3)$ & $0(0)$ & -3 \\
\hline Other & $1(0.1)$ & $0(0)$ & $1(1)$ & 1 \\
\hline Lack of Privacy & $35(2)$ & $34(53)$ & $5(3)$ & -50 \\
\hline Uncovered during delivery or examination & $17(0.9)$ & $31(48)$ & $4(3)$ & -45 \\
\hline No screens blocking view during delivery or examination & $16(0.8)$ & $29(45)$ & $0(0)$ & -45 \\
\hline Non-dignified care & $121(6)$ & $34(54)$ & $7(5)$ & -49 \\
\hline Shouted at & $35(2)$ & $24(38)$ & $0(0)$ & -38 \\
\hline Scolded & $90(5)$ & $176(25)$ & $6(4)$ & -21 \\
\hline Threatened to withhold services & $1(0.1)$ & $1(2)$ & $0(0)$ & -2 \\
\hline Called by insulting name & $3(0.2)$ & $0(0)$ & $0(0)$ & 0 \\
\hline Laughed at or scorned & $3(0.2)$ & $0(0)$ & $0(0)$ & 0 \\
\hline Other & $12(0.6)$ & $1(2)$ & $1(1)$ & -1 \\
\hline Abandonment of care & $147(8)$ & $33(52)$ & $21(14)$ & -38 \\
\hline While in Labor & $104(5)$ & $22(34)$ & $7(5)$ & -29 \\
\hline While Delivering & $60(3)$ & $12(19)$ & $11(7)$ & -12 \\
\hline While experiencing a complication & $1(0.1)$ & $0(0)$ & $0(0)$ & 0 \\
\hline After delivery & $2(0.1)$ & $0(0)$ & $2(1)$ & 1 \\
\hline Other & $5(0.3)$ & $0(0)$ & $1(1)$ & 1 \\
\hline Detention in facilities & $4(0.2)$ & $1(2)$ & $1(1)$ & -1 \\
\hline
\end{tabular}

$85.1 \%$ from baseline. Additionally, manifestations of non-dignified care such as the woman not being welcomed in a kind and gentle manner, provider not introducing themselves to the patient, and not calling women by their name, were much less frequent during the evaluation phase than at baseline.

\section{Enabling factors for success}

We strongly believe that the participatory process and sustained engagement around reducing disrespect and abuse during childbirth contributed to substantial changes in the culture of the study facility, improvement of intermediary outcomes, and, potentially, to 
Table 2 Observed disrespect and abuse

\begin{tabular}{|c|c|c|c|}
\hline Type of Disrespect and Abuse observed & $\begin{array}{l}\text { Baseline } \\
N=208 \\
n(\%)\end{array}$ & $\begin{array}{l}\text { Evaluation } \\
N=459 \\
n(\%)\end{array}$ & $\begin{array}{l}\text { Difference between } \\
\text { Evaluation and Baseline } \\
\%\end{array}$ \\
\hline \multicolumn{4}{|l|}{ Physical Abuse } \\
\hline Fundal pressure applied & $7(3.4)$ & $1(0.2)$ & -3.2 \\
\hline Lack of anesthesia for episiotomy & $9(4.3)$ & $0(0.0)$ & -4.3 \\
\hline \multicolumn{4}{|l|}{ Non-Consented Care } \\
\hline Lack of consent for first examination & $177(85.1)$ & $0(0.0)$ & -85.1 \\
\hline Lack of consent for vaginal examination & $170(81.7)$ & $0(0.0)$ & -81.7 \\
\hline \multicolumn{4}{|l|}{ Non-Confidential Care } \\
\hline Examination performed in way or setting that other clients could hear & $11(5.4)$ & $42(9.2)$ & 3.8 \\
\hline $\begin{array}{l}\text { Health providers discussed mother's private health information in a way } \\
\text { or setting that others could hear }\end{array}$ & $42(20.2)$ & $143(31.2)$ & 11.0 \\
\hline \multicolumn{4}{|l|}{ Lack of Privacy } \\
\hline No partitions separating beds & $179(86.1)$ & $118(25.7)$ & -60.4 \\
\hline Partitions did not give privacy/not adequately closed to provide privacy & $76(36.5)$ & $199(43.4)$ & 6.9 \\
\hline Mother not well covered & $133(63.9)$ & $14(3.1)$ & -60.8 \\
\hline \multicolumn{4}{|l|}{ Non-Dignified Care } \\
\hline Mother not welcomed in a kind and gentle manner & $51(24.5)$ & $0(0)$ & -24.5 \\
\hline Mother not told where to go in antenatal ward & $28(13.5)$ & $0(0)$ & -13.5 \\
\hline Delivering service provider did not congratulate the mother after birth & $55(26.3)$ & $7(1.5)$ & -24.8 \\
\hline Provider did not introduce herself to mother & $194(93.3)$ & $55(12.0)$ & -81.3 \\
\hline Mother not cleaned after birth and third stage of labor & $33(26.1)$ & $3(0.7)$ & -25.4 \\
\hline Mother not called her name throughout interactions & $123(60.0)$ & $1(0.2)$ & -59.8 \\
\hline No bed ready for mother & $113(54.3)$ & $96(20.9)$ & -33.4 \\
\hline Bed provided to mother was not clean & $135(65.9)$ & $6(1.3)$ & -64.6 \\
\hline No pad provided to the mother after birth & $17(8.2)$ & $439(95.6)$ & 87.4 \\
\hline
\end{tabular}

remarkable declines in incidence of disrespect and abuse.

In an assessment of the causes and manifestations of disrespect in hospital settings, Leape et al. stated that while the "origins of disrespectful behavior may reside in the personality characteristics of individuals and their responses to stressful environments," the "expression [of disrespectful behavior] is learned behavior and it thrives in a culture that tolerates and supports disrespect" [9]. Therefore, Leape argues, the elimination of disrespectful behavior requires an organizational culture change. Through the package of discrete interventions and sustained engagement of providers and administrators, the program initiated an open dialogue around the critical issue of D\&A and demonstrated that cultural change is possible. The results presented here indicate that important progress towards establishing respectful care as a cultural norm can be achieved in a relatively short period.

Beyond the specific intervention activities, this change in culture was facilitated by the leadership of several key champions within the study facility, including the hospital matron, head of the department of obstetrics and gynecology, the maternity ward matron, and the members of the technical working group. By modeling respectful care, making themselves available to participate in all interventions, and ensuring that dialogue around disrespect and abuse was included in all department meetings, these key staff demonstrated a commitment to respectful maternity care as a core institutional value and were instrumental to the changes observed.

Over the course of the program, our staff observed a notable shift in organizational culture and the pride the facility took in its achievements was palpable. Just as provider burnout, structural abuse, and individual biases may create reinforcing patterns of abuse $[10,11]$, we believe that empowered women, satisfied providers and patients, and strong communication can create a virtuous reinforcing cycle which fosters individual and institutional commitments to promote and embody respectful care. Additional insight into the enabling factors for the observed successes could come from the women who sought care at the study facility, although this was not included in 
our study. In particular, qualitative interviews with women who gave birth at the study facility both before and after the intervention could provide rich, detailed information about how and why their experiences differed.

The culture shift documented here is a necessary but insufficient step to ensuring respectful maternity care. In the absence of significant investments in the health system, many structural contributors to disrespect and abuse remained unchanged. Staff perceptions of the sufficiency of human resources and the availability of necessary supplies and medications was not modified from baseline to evaluation. While some observed and reported manifestations of disrespect and abuse, such as non-consented care, were nearly eliminated from baseline to evaluation, others that are directly impacted by structural and human resource deficiencies did not improve over the course of implementation. These included the prompt attention and attendance to patients from providers, privacy during care, and availability of necessary supplies and sufficient clean beds for patients. Thus, while the culture change achieved through this study is a positive shift, ensuring that all women receive dignified and respectful care will require much more, especially a commitment from the entire health system to invest in the improvement of quality of care.

\section{Conclusions}

The results presented here demonstrate that disrespect and abuse is not an intractable problem. By naming D\&A and shining a continuous light on the problem, the culture and norms at the facility began to change and the provision of respectful maternity care became an institutional priority. Through a multilevel participatory process, a low-cost package of interventions, and the establishment of champions at the facility, the program affected positive changes in provider job satisfaction, the relationships between providers and patients and providers and administrators, and in women's satisfaction with care. These interventions are among the first in Tanzania and elsewhere to demonstrate an effect in reducing D\&A, and there is widespread interest in scaling them in the program municipality and beyond. We believe that the enabling factors identified in this paper, as well as the specific interventions employed, have the strong potential to reduce disrespect and abuse and promote a culture of respectful care in other large hospitals in the region, however, a larger and more robust evaluation will be needed to determine their true impact and generalizability.

\section{Acknowledgements}

The study team extends their appreciation to the providers and administrators at the study hospital and district municipality, as well as the Tanzanian Ministry of Health and Social Welfare for their support and partnership. We also thank all of the women who shared their stories and time with us. This project was made possible through generous support from The John and Katie Hansen Family Foundation and from the Bill \&
Melinda Gates Foundation through Grant \#01065000621 for the Maternal Health Task Force in the Women and Health Initiative at the Harvard T. H. Chan School of Public Health.

\section{Authors' contributions}

All authors contributed to the conceptualization and design of the study. HLR drafted the manuscript and KPM and AL provided critical inputs. All authors read and approved the final manuscript.

\section{Competing interests}

The authors declare that they have no competing interests.

\section{Ethics approval and consent to participate}

Informed consent was sought from all women before direct observations and community follow-up postpartum interviews. Providers gave informed consent to be interviewed, and the study facility provided written informed consent for the conduct of direct observations. Participants in the Open Birth Days intervention were informed that participation was voluntary and would in no way impact the care that they would receive, at present or in the future. This study received approval from the Institutional Review Board of the Tanzanian National Institute of Medical Research and exempt status from the Harvard T.H. Chan School of Public Health Institutional Review Board. All research participants provided written or verbal informed consent.

\section{Author details}

${ }^{1}$ Women and Health Initiative, Department of Global Health and Population, Harvard T.H Chan School of Public Health, Boston, MA, USA. ${ }^{2}$ Ariadne Labs at Brigham and Women's Hospital and the Harvard T.H. Chan School of Public Health, Boston, MA, USA. ${ }^{3}$ Management and Development for Health, Dar es Salaam, Tanzania. ${ }^{4}$ Africa Academy for Public Health, Dar es Salaam, Tanzania.

${ }^{5}$ Boston University School of Public Health, Boston, MA, USA.

Received: 22 January 2016 Accepted: 19 May 2016

Published online: 18 July 2016

\section{References}

1. Ratcliffe H, Sando D, Lyatuu G, Emil F, Mwanyika-Sando M, Chalamilla G, Langer A, McDonald K. Mitigating disrespect and abuse during childbirth in Tanzania: An exploratory study of the effects of two facility-based interventions in a large public hospital. Reproductive Health; 2016.

2. Abuya T, Ndwiga C, Ritter J, Kanya L, Bellows B, Binkin N, et al. The effect of a multi-component intervention on disrespect and abuse during childbirth in Kenya. BMC Pregnancy Childbirth. BMC Pregnancy Childbirth. 2015;15: 224.

3. White Ribbon Alliance. Respectful Maternity Care: The Universal Rights of Childbearing Women. 2011. Available from: http://whiteribbonalliance.org/ wp-content/uploads/2013/10/Final_RMC_Charter.pdf. Accessed 12 Feb 2013

4. Sando D, Kendall T, Lyatuu G, Ratcliffe H, McDonald K, Mwanyika-Sando M, et al. Disrespect and abuse during childbirth in Tanzania: are women living with HIV more vulnerable? J Acquir Immune Defic Syndr. 2014;67 Suppl 4: S228-34.

5. Kruk ME, Kujawski S, Mbaruku G, Ramsey K, Moyo W, Freedm LP. Disrespectful and abusive treatment during facility delivery in Tanzania: a facility and community survey. Health Policy Plan. 2014;1-8. doi:10.1093/ heapol/czu079.

6. Sando D, Ratcliffe H, McDonald K, Spiegelman D, Lyatuu G, MwanyikaSando $\mathrm{M}$, et al. The prevalence of disrespect and abuse during facility-based childbirth in urban Tanzania. In Press.

7. Ratcliffe H. Creating an evidence base for the promotion of respectful maternity care. Harvard School of Public Health; 2013. Available from: http:// wordpress.sph.harvard.edu/mhtf-2/wp-content/uploads/sites/32/2014/11/ EvidenceBaseForRMC_MHTF_Rep_2013.pdf. Accessed 29 Apr 2015.

8. Fonn S, Mtonga AS, Nkoloma HC, Bantebya Kyomuhendo G, da Silva L, Kazilimani $\mathrm{E}$, et al. Health providers' opinions on provider-client relations: results of a multi-country study to test Health Workers for Change. Health Policy Plan. 2001;16 Suppl 1:19-23.

9. Leape LL, Shore MF, Dienstag JL, Mayer RJ, Edgman-Levitan S, Meyer GS, et al. A culture of respect, Part 2: creating a culture of respect. Acad Med. 2012;87:853-8. 
10. Leape LL, Shore MF, Dienstag JL, Mayer RJ, Edgman-Levitan S, Meyer GS, et al. A culture of respect, Part 1: The nature and causes of disrespectful behavior by physicians. Acad Med. 2012;87:845-52.

11. Ruotsalainen J, Verbeek J, Marine A, Serra C. Preventing occupational stress in healthcare workers (Review). Cochrane Database Syst Rev. 2015. Issue 4. Art. No.: CD002892. doi:10.1002/14651858.CD002892.pub5.

Submit your next manuscript to BioMed Central and we will help you at every step:

- We accept pre-submission inquiries

- Our selector tool helps you to find the most relevant journal

- We provide round the clock customer support

- Convenient online submission

- Thorough peer review

- Inclusion in PubMed and all major indexing services

- Maximum visibility for your research

Submit your manuscript at www.biomedcentral.com/submit 